\title{
The Efficiency of Laparoscopic Sleeve Gastrectomy in Improving Patients with Type II Diabetes Mellitus
}

\author{
A.A.Salem, E.A.Abd El-Mabood, A.M.Nowar, and M.A.Abulyazeed \\ General Surgery, Dept., Faculty of Medicine, Benha Univ., Benha, Egypt \\ E-Mail:Abulyazeed56@gmail.com
}

\begin{abstract}
Background Laparoscopic Sleeve gastrectomy (LSG) was utilised in super-obese patients with at least two serious co-morbidities as a first step of a multi-phase treatment. The objective of this research is to assess the effectiveness of laparoscopic sleeve gastrectomy in treating and improving type II diabetes mellitus. Methods: this research included 40 patients with diabetes, morbid obesity and laparoscopic pulmonary gastrectomy and post operational follow-up for 6-12 months, by measuring serum haemoglobin A1c (HbA1c) and fasting glucose blood for their condition. Results: $67.5 \%$ of our patients analysed were female and the remaining $32.5 \%$ were male and their age varied from 21 to 44 years with average age $31.3 \pm 8.1$. Only one individual reported of postoperative haemorrhage without surgical death. With respect to connections between HbA1c, Body Weight and BMI, before and $6 \& 12$ months postoperative data, there were significant connections between BMI at 6-12 months postoperative and HbA1c at 12 months postoperative. At the three observations "preoperative and 6 \& 12 months postoperative," however, no significant association was identified between body weight and HbA1c results, Conclusion: LSG is more useful for obesity and diabetes resolution in morbidly obese diabetic patients. However, more observation is still necessary to determine the effects of LSG on hypertension and hyperlipidemia.
\end{abstract}

Keywords: laparoscopic, sleeve; gastrectomy; diabetes mellitus; DM.

\section{Introduction}

Obesity A chronic metabolic disorder that affects adults and children across the globe. Obesity is acknowledged to be the principal risk factor for many non-communicable illnesses, including type 2 diabetes [1]. It has become one of the major cause of mortality.

Diabetes mellitus (DM) is a chronic, persistent hyperglycemia metabolic disease. It may be because of the decreased production of insulin, resistance to insulin peripheral activities or both. According to the IDF, roughly 415 million persons aged between 20 and 79 years had diabetes mellitus in 2015[2].

Laparoscopic sleeve gastrectomy utilised in multistage surgery as the initial step of at least two serious co-morbidities in superobese patients. More than 50 percent of their patients have exhibited remission of their co-morbidities two years following sleeve gastrectomy. The technique has gained appeal since it was introduced because of its simplicity, excellent weight loss and less problems. Despite the aforementioned, there have been particular issues. The effects of Laparoscopic Sleeve Gastrectomy on several components of metabolic syndrome have recently been shown by the population of patients with T2DM. Although the biological processes underpinning insulin sensitivity and $B$ cell activity caused by the procedure remain unclear, the surgery is largely designed to targeted the pathophysiology of this disease[3].

The purpose of this research was to evaluate the efficacy of laparoscopic sleeve gastrectomy in diabetes management and improvement. Mellitus

\section{Patients and methods}

This This prospective observer research was carried out on 40 patients with Type 2 D.M morbid obesity and laparoscopic pulp gastrectomy, and 6 and 12 months post-operatively monitored at Benha
University Hospitals for a period of 28 months from January 2016 to May 2018.

After the consent of the local research council, laparoscopic sleeved gastrectomy was performed to evaluate the short- and long-term effects on glucose homeostasis of the surgery. All registered patients were thoroughly advised and given an informed consent after their knowledge of the research objectives Inclusion Criteria:

- Study age: 18 years to 65 years.

- Study qualifying genders: (27 females \& 13 males).

- Obesity. Type II Diabetes Mellitus Patients

- BMI $35 \mathrm{Kg} / \mathrm{m} 2$ in length.

- Generally healthy people without serious systemic disease and suitable for surgery

- Selected individuals are treated regularly for either insulin or oral hypoglycemic diabetes miletus for at least one year before the procedure.

- Things are picked from patients with non-sweeteaters.

- Failed other measurements of weight reduction for at least six months before the operation

- People who are psychologically stable.

- Accessibility and readiness to participate in follow-up visits.

Criteria for exclusion:

- Earlier abdominal operation with the exception of laparoscopic operations.

- Severe COPD, the form of obstructive pulmonary disease characterised by continuously inadequate airflow. It usually becomes worse with time. The major symptoms are shortness of breath, cough and sputum.

- Cardiac unsuitable anaesthesia patients.

- Obesity related to endocrine dysfunction 
clinically diagnosed.

- Things with liver functions hindered.

- Malignant illness history.

- Pregnant or pregnancy planning during study enrollment.

- Inability to get informed approval.

- Follow-up inaccessibility.

- The patient's inability or reluctance to modify post-operative lifestyles

- Alcohol or drug or other substance dependence.

- Antagonistic families or unfriendly home patients.

All the patients got thorough training before to surgery in the form of written documents, films and group talks regarding LSG's risks, advantages and alternatives. These tools were offered for patients and their families in order to evaluate their desire to help their patients postoperatively.

All patients had a comprehensive history, comprehensive clinical examination, Workup in the laboratory: Full blood count, serum calcium, fasting blood glucose, haemoglobin, creatinine, fasting lipid profile, thyroid profile, serum cortisol and urinary analysis (for glycosuria and proteinuria). Abdominal U/S to demonstrate stones and hepatomegaly of gallbladder. Cardiological evaluation by: electrocardiogram resting and echocardiography. And Respiratory evaluation by: X-ray chest, respiratory function test.

\section{Operational technology:}

Positioning and port positioning:

The patient is placed in a reversed trendlenberg posture with split legs, after induction of anaesthesia and administration of preventive antibiotics, with elastic legs as a preventative DVT measure.

After the patient's skin was sterilised, insufflation of the abdomen began using a closed Veras needle in the mid-clavicular area of the left subcostal region. Introduction of the visi-port optical 5-12 $\mathrm{mm}$ with inside $10 \mathrm{~mm} 30$ digrees, followed by examination of the breathing site and removal of the visual insufflation needle and diagnostic investigation by laparoscopy

A further $10 \mathrm{~mm}$ port is put in the right midclavicular subcostal area beneath vision as well as two $5 \mathrm{~mm}$ ports in the epigastric left paramedian area and finally at the left anterior axilar subcostal line.

A fifth $5 \mathrm{~mm}$ subxyphoid trocar is inserted as a liver after 11 minutes from the commencement of the procedure retractor Fig. (1).

\section{Procedure Steps:}

We started by dissection of the greater omentum with the Ligasure shear by creating a window to the lesser sac at a point in the middle of the greater curvature of the stomach Fig. $(2,3)$ then dividing the omentum upwards until the gastro-splenic ligament is reached Fig. (4), at this point another $5 \mathrm{~mm}$ trocar is introduced as a liver retractor Fig. (5) and we continued dividing the gastro-splenic ligament Fig. (6).

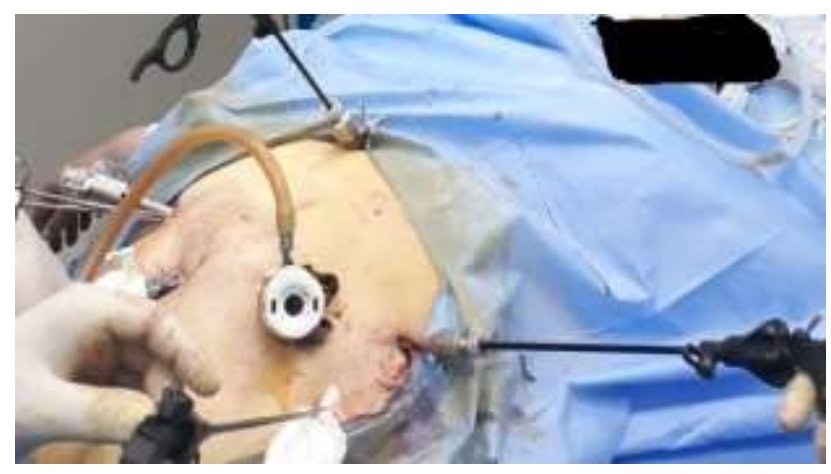

Fig. (1) Trocars for laparoscopic sleeve gastrectomy.

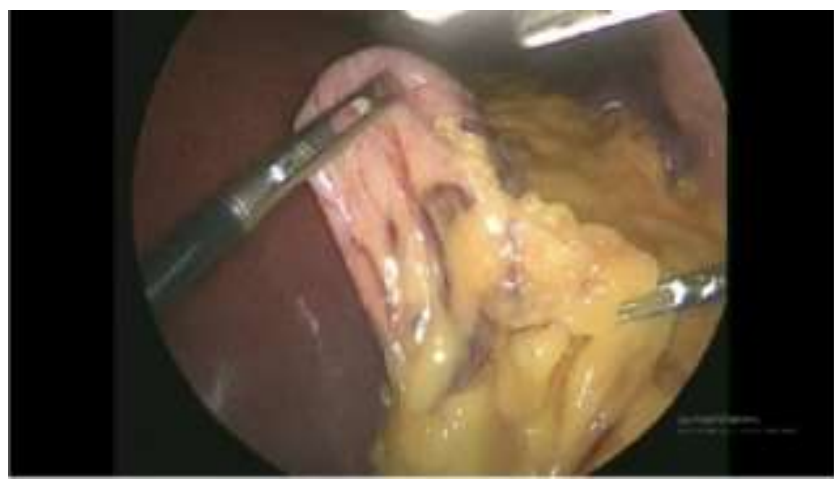

Fig. (2) The start point of dissection. 


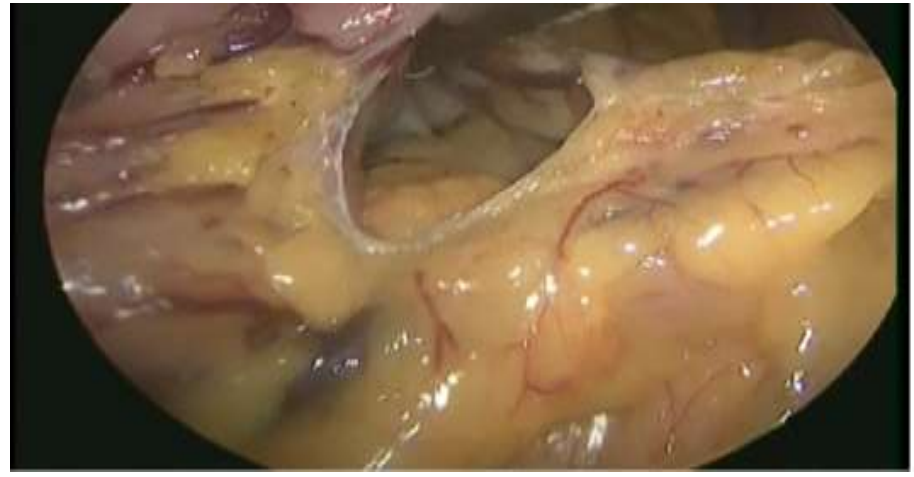

Fig. (3) Creating a window to the lesser sac.

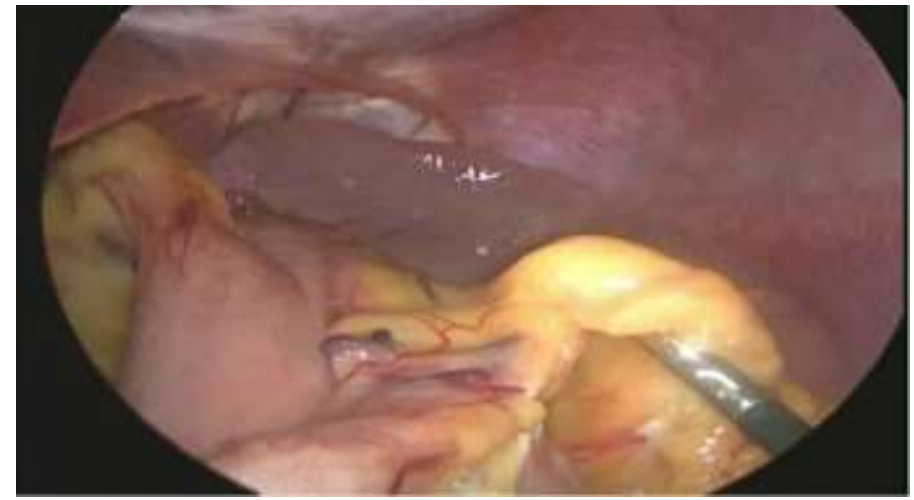

Fig. (4) Upward dissection until the gastrosplenic ligament is reached.

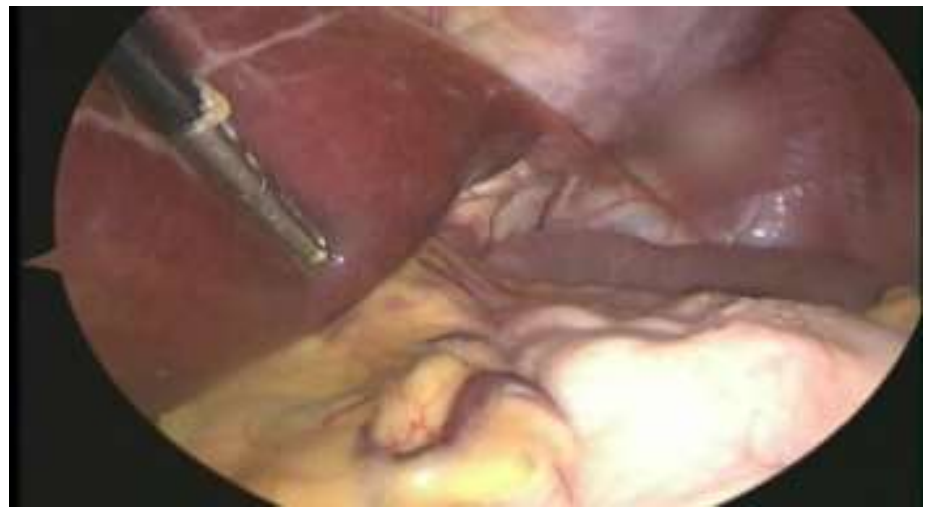

Fig. (5) Trocar introduced as a liver retractor.

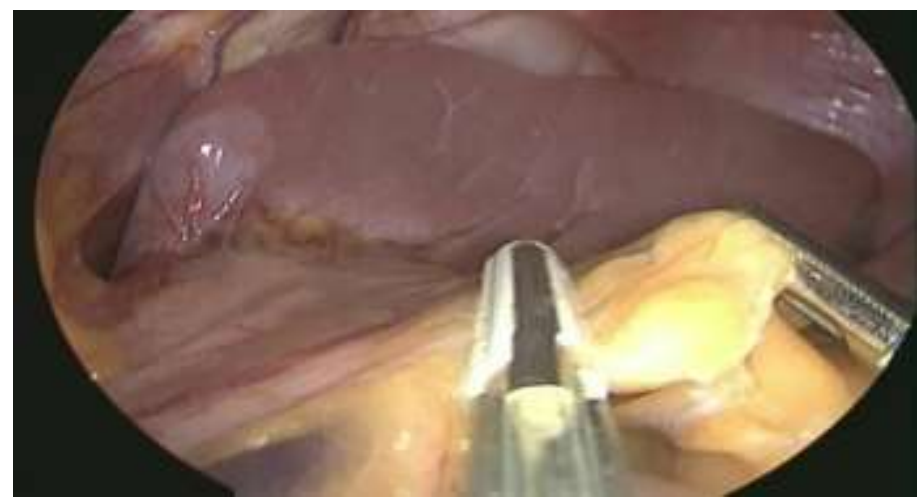

Fig. (6) Dividing the gastrosplenic ligament. 
The angle of Hid is identified and dissected free from the left crus and all the fat was removed over the gastroesophageal junction to identify it Fig. (7).

Then the greater curvature dissection is continued from the start point distally until about $4 \mathrm{~cm}$ from the pylorus Fig. (8) After that we proceeded lysis of all adhesions in the lesser sac and the gastro-pancreatic attachments Fig. (9).

The anaesthiest was asked to introduce 32-Fr bougie under laparoscopic vision to ensure patency of the stomach during stappling and we directed it to the pylorus using a soft bowel grasper Fig. (10).

At the level of the crow's foot distal to the incisura angularis about $5 \mathrm{~cm}$ proximal to the pylorus we started our first fire stapler using a green Covidien cartridge and firing was done after ensuring free mobility of the boogie Fig. (11), the second stapler is aligned with the first one using blue Covidien cartridge Fig. (12) And then fired after inspection of both anterior and posterior walls of the stomach and making sure there was no twisting of the lumen and that equal traction is applied Fig. (13) And then stapling continued in the same manner until the body is completely divided.

After completion of the procedure the anesthesiologist performed a leakage test using diluted methylene blue under laparoscopic vision and gentle clamping of distal duodenum and then removed the bougie carefully. Hemostatic titanium clips are applied to the bleeding points on the stapling line to ensure hemostasis Fig. (14).

The excised stomach is then retrieved through the site of the stapler port under vision using a retrieval bag to prevent contamination of the wound.

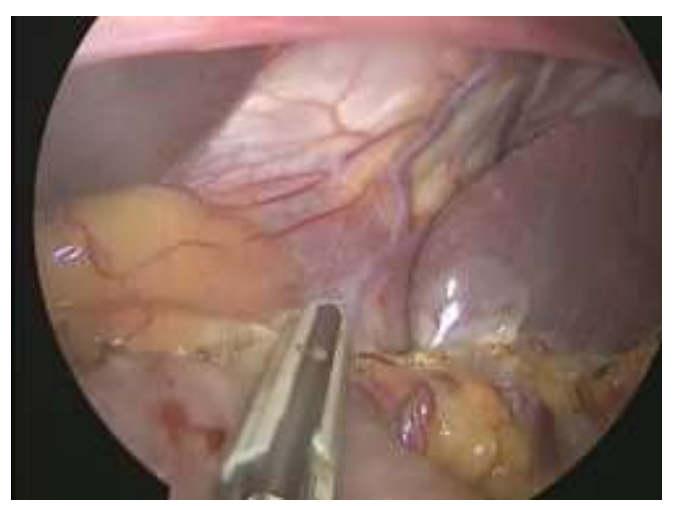

Fig. (7) Dissection of the left crus and gastroesophageal junction

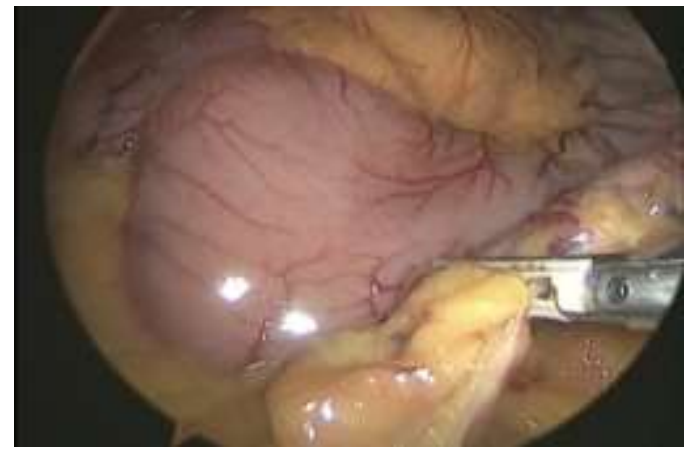

Fig. (8) Continuing the distal dissection of the greater curvature

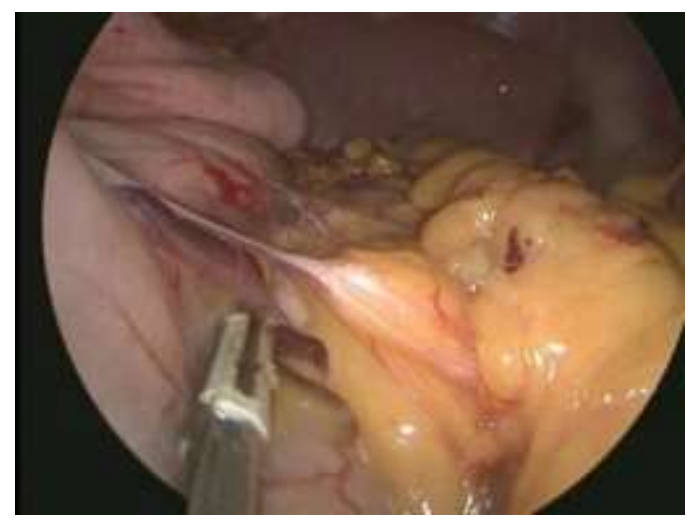

Fig. (9) Adhesolysis of posterior wall of the stomach 


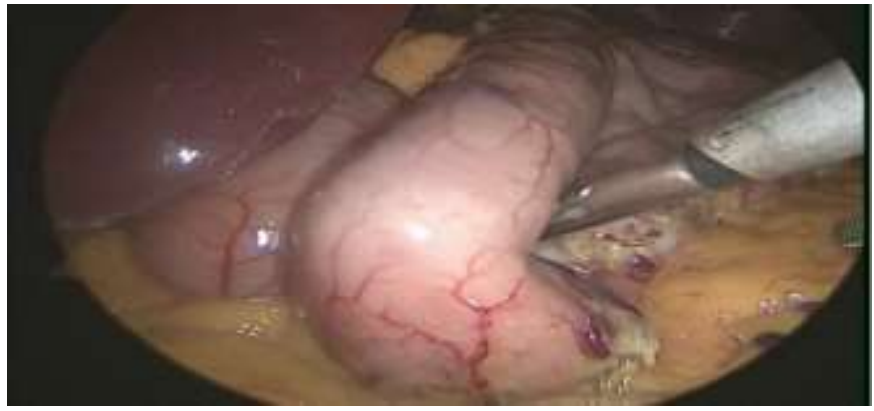

Fig (10): Introduction of the bougie under laparoscopic vision

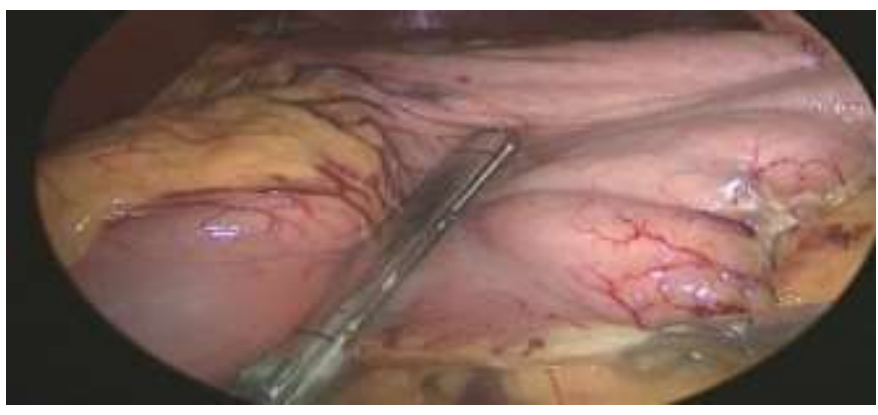

Fig (11): the first stapler firing point

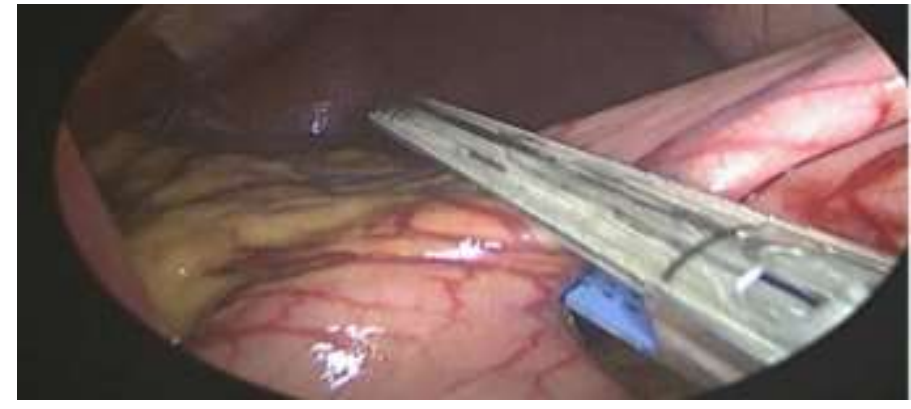

Fig (12): The second stapler aligned with the first one

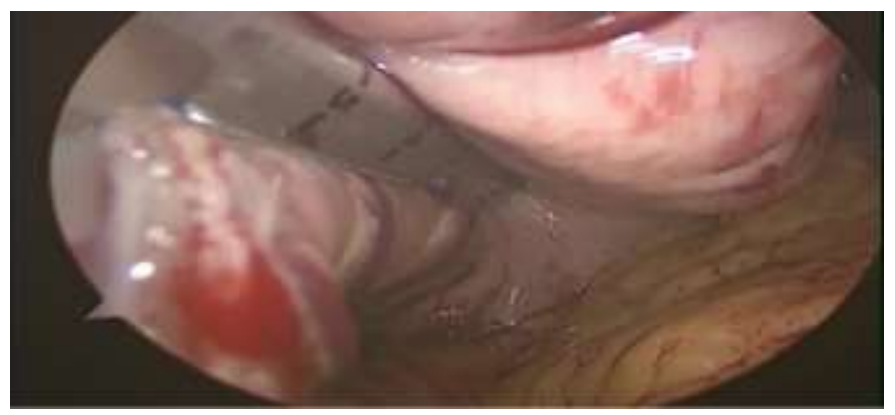

Fig (13): inspection of posterior wall before firing each cartridge

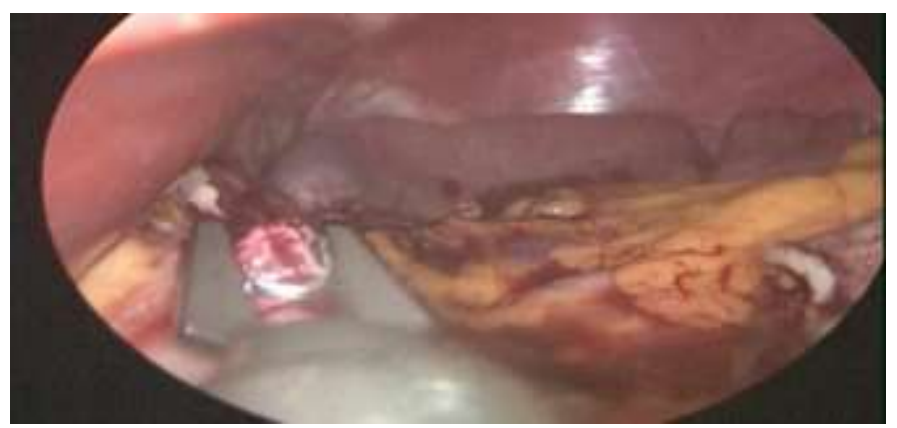

Fig (14): Hemostatic clips applied to ensure hemostasis 


\section{Closure}

Inspection of the stapling line is done and a $20-\mathrm{Fr}$ tube drain is inserted under vision thought the most left lateral trocar and the tube drain was fixed using silk suture. The rest of port-sites were closed using 3-0 Vicryl interrupted sutures.

\section{Postoperative Data:}

- Follow up weight documentation and body mass index preoperatively, 6 and 12 months after the operation

- Fasting blood sugar 6 and 12 months after the operation.

- HbA1c in blood 6 and 12 months after the operation.

\section{Statistical Analysis}

The data was coded and entered using the statistical package for social science (SPSS) version 22. The data was summarized using descriptive statistics: mean median, standard deviation and range for quantitative variables. The comparison between the preoperative and 6 month of the studied variables were tested using Wilcoxon signed ranks test and correlations. P-values less than or equal to 0.05 were considered statistically significant. Graphs were carried out using Microsoft office Excel to show the differences in preoperative and 6,12 months follow up and correlations between the variables of the study.

\section{Results}

In this study, $67.5 \%$ of studied group were females while the rest $32.5 \%$ were males and their ages were rnged between $21-44$ years with mean age 31.3 \pm 8.1 years. $47.5 \backslash \%$ of our patients were complaining of both peripheral and central obesity while the othes were $35.0 \%$ central only and $17.5 \%$ peripheral only. 27.5 of them were on insulin therapy preoperative table (1)

Table (1) Gender and age distribution of patients.

\begin{tabular}{lll}
\hline Age (in years): & & \\
Mean \pm SD & $31.3 \pm 8.1$ & \\
Range & $21-44$ & Percent $(\%)$ \\
Gender & No & $32.5 \%$ \\
$-\quad$ Male & 13 & $67.5 \%$ \\
$-\quad$ Female & 27 & $17.5 \%$ \\
Distribution of obesity: & 7 & $35.0 \%$ \\
$-\quad$ Peripheral & 14 & $47.5 \%$ \\
$-\quad$ Central & 19 & \\
$-\quad$ Both & & $67.5 \%$ \\
Duration of DM (years) & 27 & $32.5 \%$ \\
$-\quad$ > 5 years & 13 & $72.5 \%$ \\
Preoperative medication: & & $27.5 \%$ \\
$-\quad$ OHG & 29 & $100 \%$ \\
\hline$\quad$ Insulin & 11 & 40 \\
\hline
\end{tabular}

Baseline (preoperative) data of our patients' body weights, BMI, their FBS and Hb A1c levles in table (2).

Table (2) Baseline biochemical characteristics of the studied patients group.

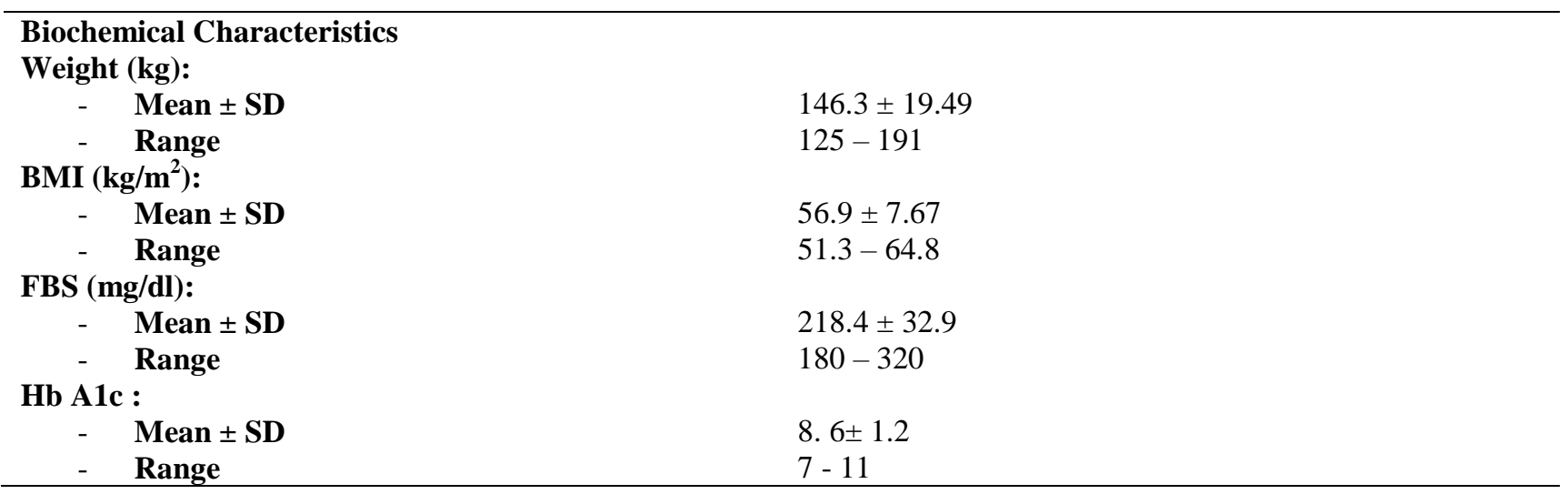

There was a statistical significant reduction in BMI of the studied group at 6 and 12 months post-operative in comparison with that at pre-operative, table (3). 
Table (3) Comparison between baseline body mass index and after $6 \& 12$ months after surgery.

\begin{tabular}{|c|c|c|c|c|c|c|}
\hline $\begin{array}{l}\text { Body } \\
\text { (BMI): }\end{array}$ & Index & At Baseline & After 6 months & After 12 months & F value & P value \\
\hline- & Mean \pm SD & $56.9 \pm 7.67$ & $48.6 \pm 5.82$ & $34.9 \pm 3.3$ & 520.33 & $\begin{array}{l}<0.001 \\
\mathrm{HS}\end{array}$ \\
\hline
\end{tabular}

There was a statistical significant reduction in body weigths of the studied group at 6 and 12 months after surgery in comparison with that at baseline table (4).

Table (4) Comparison between baseline weights and after $6 \& 12$ months.

\begin{tabular}{cllllll}
\hline Weight $($ WT): & & At Baseline & After 6 months & After 12 months & F value & P value \\
\hline $\begin{array}{c}\text { Mean } \\
\text { SD }\end{array}$ & \pm & $146.3 \pm 19.49$ & $101.4 \pm 11.13$ & $89.87 \pm 10.26$ & 787.15 & $<0.001$ \\
\hline
\end{tabular}

There was a statistical significant improvement in FBS of the studied group at 6 and 12 months post-operative in comparison with that at pre-operative table (5).

Table (5) Comparison between baseline fasting blood sugar and after 6 \& 12 months.

\begin{tabular}{|c|c|c|c|c|c|}
\hline $\begin{array}{l}\text { Fasting Blood Sugar } \\
\text { (WT): }\end{array}$ & At Baseline & After 6 months & After 12 months & F value & $P$ value \\
\hline - $\quad$ Mean \pm SD & $218.4 \pm 32.9$ & $127.4 \pm 9.22$ & $105.6 \pm 16.4$ & 492.87 & $\begin{array}{l}<0.001 \\
\text { HS }\end{array}$ \\
\hline
\end{tabular}

There was a statistical significant improvement in $\mathrm{Hb} \mathrm{A} 1 \mathrm{c}$ of the studied group at 6 and 12 months post-operative in comparison with that at pre-operative table (6).

Table (6) Comparison between baseline Hemoglobin A1c and after 6 \& 12 months post-operative.

\begin{tabular}{cccccc}
\hline $\begin{array}{l}\text { Hemoglobin A1c } \\
\text { (Hb A1c): }\end{array}$ & At Baseline & After 6 months & After 12 months & F value & P value \\
\hline Mean + SD & $8.6 \pm 1.2$ & $6.82 \pm 0.67$ & $6.14 \pm 0.76$ & 78.51 & $<0.001$ \\
\hline
\end{tabular}

There was a statistical significant correlation between Baseline body weight and baseline FBS. Also, there was a significant correlation between Body weight and FBS at 6 and 12 postoperative table (7).

Table (7) Correlation between weight and fasting blood sugar.

\begin{tabular}{llllllll}
\hline Weight (WT): & \multicolumn{2}{c}{ Baseline Wt. } & \multicolumn{2}{c}{ After 6 months Wt. } & \multicolumn{2}{c}{ After 12 months Wt. } \\
& & r - value & p - value & r - value & p - value & r - value & p - value \\
\hline- & Baseline FBS & .046 & $0.004^{*}$ & 0.639 & $<0.001^{*}$ & 0.558 & $<0.001^{*}$ \\
- & FBS After 6 months & 0.019 & 0.912 & 0.417 & $0.007^{*}$ & 0.519 & $<0.001^{*}$ \\
- & FBS after 12 months & 0.094 & 0.563 & 0.612 & $<0.001^{*}$ & 0.6119 & $<0.001^{*}$ \\
\hline
\end{tabular}

There was a statistical significant correlation between BMI and FBS at $6 \& 12$ month postoperative table (8).

Table (8) Correlation between BMI and fasting blood sugar.

\begin{tabular}{llllllll}
\hline Weight (WT): & \multicolumn{2}{c}{ Baseline BMI. } & \multicolumn{2}{c}{ After 6 months BMI. } & \multicolumn{2}{c}{ After 12 months BMI. } \\
& & r - value & p - value & r - value & p - value & r - value & p - value \\
\hline - & Baseline FBS & -0.108 & 0.511 & 0.307 & 0.054 & 0.245 & 0.127 \\
- & FBS After 6 months & -0.123 & 0.449 & 0.599 & $<0.001 *$ & 0.742 & $<0.001 *$ \\
- & FBS after 12 months & -0.019 & 0.908 & 0.633 & $<0.001 *$ & 0.655 & $<0.001 *$ \\
\hline
\end{tabular}

There was no significant correlations were observed between body weights and $\mathrm{Hb} \mathrm{A} 1 \mathrm{c}$ findings at the 3 obesrvation "preoperative and $6 \& 12$ months postoperative" table (9). 
Table (9) Correlation between weight and HbA1c.

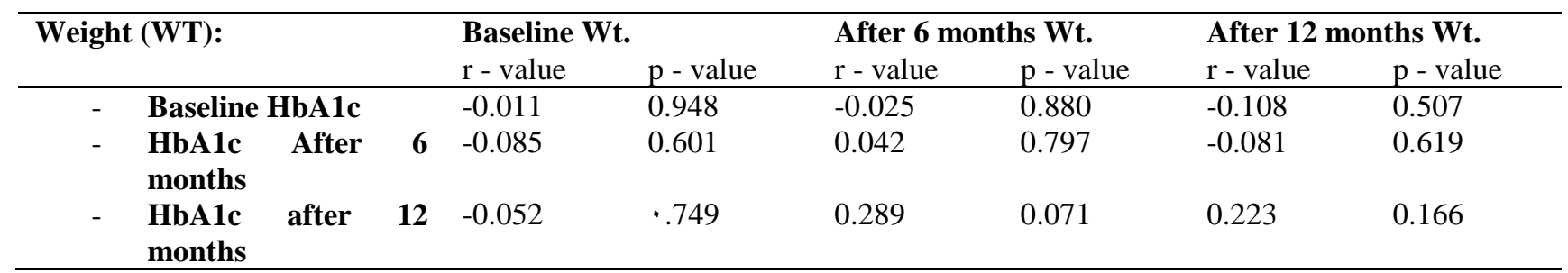

There was a significant correlations between BMI at 6 and 12 months postoperative and HbA1c 12 month postoperative. table 10

Table (10) Correlation between BMI and HbA1c.

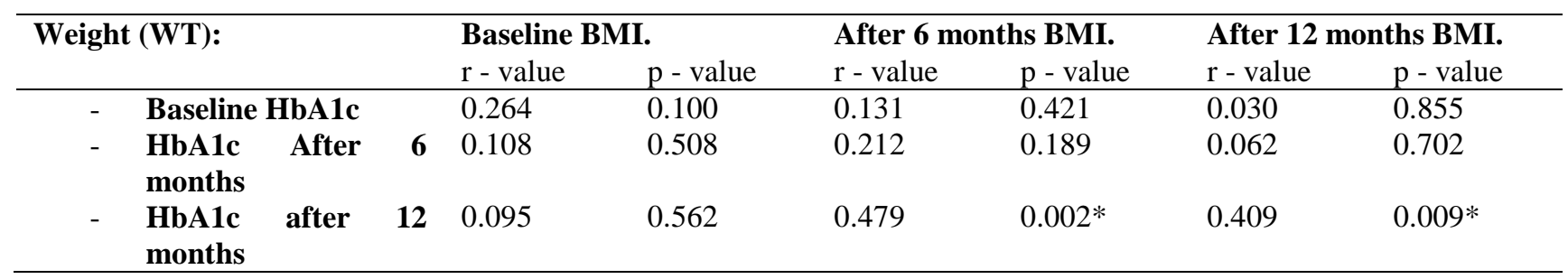

Only one patient complained postoperative bleeding. While no operative mortality were reported. table 11

Table (11) Incidence of postoperative Complications and operative mortality.

\begin{tabular}{|c|c|c|}
\hline Variable & No & Percent (\%) \\
\hline Postoperative Complications: & 1 & $2.5 \%$ \\
\hline $\begin{array}{ll}- & \text { Yes } \\
- & \text { No }\end{array}$ & 39 & $97.5 \%$ \\
\hline Postoperative Complications: & 1 & \\
\hline $\begin{array}{ll}\text { - } & \text { Hemorrhage } \\
\text { - } & \text { Leakage }\end{array}$ & 0 & \\
\hline Operative Mortality: & 0 & $0.0 \%$ \\
\hline $\begin{array}{ll}- & \text { Yes } \\
- & \text { No }\end{array}$ & 40 & $100.0 \%$ \\
\hline
\end{tabular}

\section{Discussion}

The The basic weight ranged from $125-191 \mathrm{~kg}$ with a mean value of: $146.3 \pm 19.49 \mathrm{~kg}$ for individuals included in our research. In contrast, baseline BMI ranged from 51.3 to $64.8 \mathrm{~kg} / \mathrm{m} 2$ with medium value: $56.9 \pm 7.67 \mathrm{~kg} / \mathrm{m} 2$. And follow-up indicated that at 6 and 12 months following surgery, their body weights and BMI were reduced significantly with a p-value $<0.001$.

According to our results, Attia has [4] reported a substantial weight loss and a decrease in BMI after LSG. In their comprehensive review, Gill et al. [5] also observed that the mean preoperative BMI was $47.4 \pm$ $7.9 \mathrm{~kg} / \mathrm{m} 2$ (31-53.5 range), based on 13 research. Significant weight loss excess was shown in 11 trials at a significant percentage of 47.3 percent \pm 19.1 percent (range 6.3-74.5 percent) with a mean follow-up of $13 \pm$ 8.1 months (range $3-36$ ). Based on 8 included studies, postoperative BMI has decreased to $35.9 \pm 6.6 \mathrm{~kg} / \mathrm{m} 2$ (range 24.6-44.7).

Morbid obese patients will have a number of physical changes after sleeve gastrectomy, including a significant long-term loss of weight (up to $80 \%$ excessive loss of weight "EWL"; about $10 \%$ lower than the "RYGB" Roux-en-Y gastric circumstances), longterm maintenance of EWL percentage, a drop in hunger, satiety, food preferential changes and an increase in energy costs[6].

With regard to the glycemic parameters "FBS and HbA1c," our results demonstrated that the results of FBS and HbA1c are significantly improved after 6 or 12 months as compared to their preoperative values.

In accordance with our results, Attia [4] said in their research that there was a considerable improvement in FBS and HbA1c "by reducing HbA1c less than 7 percent" in the postoperative phase. Similarly, following surgery, Schauter et al.,[7] who discovered 150 obese individuals with uncontrolled type $2 \mathrm{DM}$ in their research, had SG, and that there was a substantial improvement in HbA 1c after surgery, and that after SG, diabetes recovery was $6 \%$ or less, as well.

Also Schauer et al., [9] and Purnell et al., [10] demonstrated that obese individuals (BMI 27 - 43) with T2D were more successful and practicable than 
rigorous medical treatment alone in lowering hyperglycemia [3].

Our findings revealed that $70 \%$ of patients demonstrated HBA1c diabetes recovery below 6.5; in $25 \%$ of patients between $6.5-7$; and in $5 \%$ of patients remaining above 7 .

In the evaluation and meta-analysis research conducted by Gill et al.[5] which reviewed 27 trials and 673 patients, it was stated that $66.2 \%$ of patients had diabetes resolved, $26.9 \%$ of patients improved and $13.1 \%$ remained constant.

The improvement of diabetes state may be explained by the substantial increase in insulin sensitivity after sleeve gastrectomy and a considerable decrease in FBS and HbA1c levels[11]. This is mostly due to the reduction in ghrelin serum levels and the rise in CCK, GIP, GLP1, and GLP 2, which are essential for diabetes resolution and metabolism regulation. Insulin resistance remission has also been connected in very obese individuals receiving bariatric surgery with serum uric acid decline[3].

With regard to the link between fbs, body weights and bmi, preoperatively acquired findings and postoperative 6 and 12 months, our results suggest that there is a substantial statistical link between body weight and baseline FBS. In addition, there was a substantial association between 6 and 12 postoperative body weight and FBS. At 6 and 12 months postoperatively, there was a statistically significant connection between BMI and FBS.

As regards the preoperative, 6 and 12 month postoperative correlations between $\mathrm{HbA1c}$, Body weight and BMI, our data demonstrated significant correlations between BMI 6 to 12 months postoperative and $\mathrm{HbA} 1 \mathrm{c} 12$ months only postoperative. At the three observations "preoperative and 6 \& 12 months postoperative" no significant associations were identified between bodily weights and $\mathrm{HbA} 1 \mathrm{c}$ readings.

With regard to postoperative complications and surgical mortality, our findings revealed that, although there were no operating fatality, only one patient complained of post-operative bleeding.The overall condition was stable and the vital statistics were steady (HR: $100 \mathrm{p} / \mathrm{m}$ and BP: 105/ 60). This patient was convectively treated and two blood units were provided.

Attia[4] claimed that there was no severe problem in their research and no operative mortality.

\section{Conclusion}

Gastrectomy (LSG) is a simple, safe and effective approach for surgery that results in a large reduction of weight without mortality and co-morbidity, with lower complications. It is not merely a weight reduction operation, but a metabolic operation that may treat most of the metabolic syndrome. Most patients with type $2 \mathrm{DM}$ have shown resolution or improvement of biochemical homeostasis of glucose and may be regarded a strategy for treating morbidly obese individuals with impaired glucose metabolism after LSG. In obese individuals, the most effective long-term method of therapy for type 2 diabetes is evaluated.

LSG is particularly helpful in the resolution of obesity and diabetes in morbidly obese diabetic patients. However, more observation is still necessary to determine the effects of LSG on hypertension and hyperlipidemia.

The favourable impact (remission and/or improvement) of LSG on diabetes and, in general, on chronic complications of the illness are required on a medium- and long-term basis. Above all, long-term outcomes will enable us to evaluate sleeve gastrectomy costs and advantages to standard medical treatment. treatments.

\section{References}

[1] D. R. Leitner, "Obesity and type 2 diabetes: Two diseases with a need for combined treatment strategies-EASO can lead the way," Obes. Facts, vol. 10, pp. 483-492, 2017.

[2] Y. Zheng, S. H. Ley, and F. B. Hu, "Global aetiology and epidemiology of type 2 diabetes mellitus and its complications," Nat. Rev. Endocrinol., vol. 14, pp. 88, 2018.

[3] M. Kheirvari , "The advantages and disadvantages of sleeve gastrectomy; clinical laboratory to bedside review," Heliyon, vol. 6,pp. e03496, 2020.

[4] R. Attia, "Role of sleeve gastrectomy in control of type 2 diabetes- a prospective clinical study," Egypt. J. Surg., vol. 38, pp. 267, 2019.

[5] R. S. Gill, D. W. Birch, X. Shi, A. M. Sharma, and S. Karmali, "Sleeve gastrectomy and type 2 diabetes mellitus: a systematic review," Surg. Obes. Relat. Dis., vol. 6, pp. 707-713, 2010.

[6] P. Sinclair, D. J. Brennan, and C. W. le Roux, "Gut adaptation after metabolic surgery and its influences on the brain, liver and cancer," Nat. Rev. Gastroenterol. Hepatol., vol. 15 10, pp. 606624, 2018.

[7] P. R. Schauer , "Bariatric surgery versus intensive medical therapy for diabetes-3-year outcomes," N. Engl. J. Med., vol. 370, pp. 2002-2013, 2014.

[8] M. Milone , "Bariatric surgery and diabetes remission: sleeve gastrectomy or mini-gastric bypass?," World J. Gastroenterol. WJG, vol. 19, pp. 6590, 2013.

[9] P. R. Schauer , "Bariatric surgery versus intensive medical therapy for diabetes-5-year outcomes," N Engl J Med, vol. 376, pp. 641-651, 2017.

[10] J. Q. Purnell, "Definitions, classification, and epidemiology of obesity," in Endotext [Internet], MDText. com, Inc., vol. 1, pp. 1-11, 2018.

[11] İ. D. Duran, N. E. Gülçelik, B. Bulut, Z. Balc1, D. Berker, and S. Güler, "Differences in calcium metabolism and thyroid physiology after sleeve gastrectomy and Roux-En-Y gastric bypass," Obes. Surg., vol. 29, pp. 705-712, 2019. 